\title{
Relativistic Current Dynamics Investigations By Proton Probing
}

\author{
M. Borghesi, ${ }^{a}$ K. Quinn, ${ }^{\text {a }}$ P. A. Wilson, ${ }^{\text {a }}$ C. A. Cecchetti, ${ }^{a}$ \\ B. Ramakrishna, ${ }^{\text {a }}$ L. Romagnani, ${ }^{\text {a }}$ G. Sarri, ${ }^{\text {a }}$ L. Lancia, ${ }^{\text {b }}$ J. Fuchs, ${ }^{b}$ \\ A. Pipahl, ${ }^{\mathrm{c}}$ T. Toncian, ${ }^{\mathrm{c}}$ O. Willi, ${ }^{\mathrm{c}}$ D. C. Carroll, ${ }^{\mathrm{d}}$ P. Gallegos, ${ }^{\mathrm{d}}$ \\ M. N. Quinn, ${ }^{\mathrm{d}}$ X. H. Yuan, ${ }^{\mathrm{d}}$ P. McKenna, ${ }^{\mathrm{d}}$ R. J. Clarke, ${ }^{\mathrm{e}}$ R. G. Evans, ${ }^{\mathrm{e}}$ \\ D. Neely, ${ }^{\mathrm{e}}$ M. Notley, ${ }^{\mathrm{e}}$ A. Macchi, ${ }^{\mathrm{f}}$ T. V. Lyseikina, ${ }^{\mathrm{g}}$ W. Nazarov, ${ }^{\mathrm{h}}$ \\ ${ }^{a}$ Department of Physics and Astronomy, Queen's University Belfast, Belfast BT7 INN, UK \\ ${ }^{b}$ Laboratoire pour l'Utilisation des Lasers Intenses, École Polytechnique, 91128 Palaiseau, France \\ ${ }^{c}$ Institut für Laser-und Plasmaphysik, Heinrich-Heine-Universität, D-40225 Düsseldorf, Germany \\ ${ }^{d}$ SUPA, Department of Physics, University of Strathclyde, Glasgow G4 ONG, UK \\ ${ }^{e}$ Central Laser Facility, Rutherford Appleton Laboratory, Chilton, Oxfordshire OX11 0QX, UK \\ ${ }^{f}$ CNR/INFM/polyLAB, Dipartimento di Fisica "E. Fermi", Pisa, Italy \\ ${ }^{g}$ Max Planck Institute for Nuclear Physics, Heidelberg, Germany \\ ${ }^{h}$ School of Chemistry, University of St. Andrews, St. Andrews KY16 9ST, UK
}

\begin{abstract}
The proton probing technique has been used to investigate the incidence of a mid- $10^{19}$ $\mathrm{W} \mathrm{cm}{ }^{-2}$ pulse with metallic wire and laminar foam targets. Electric fields $\sim 10^{10} \mathrm{~V} \cdot \mathrm{m}^{-1}$ are measured on the surface of the $125 \mu \mathrm{m}$-diameter wire in the wake of the laser interaction as it charges and discharges within a $20 \mathrm{ps}$ temporal window, whilst the employment of a novel experimental technique permits the observation of the propagation of a charging front at $\sim c$ away from the point of interaction. In the foam shots, meanwhile, the behaviour of the hot electrons generated by the interaction pulse is probed inside the target. Evidence of electric inhibition effects and filamentation is found.
\end{abstract}

Keywords: short-pulse, high-intensity laser-matter interactions, ion, proton acceleration, return current, electron escape, electron transport, electric inhibition, filamentation, instability

PACS: $41.75 . \mathrm{Jv}, 52.38 . \mathrm{Kd}, 52.57 . \mathrm{Kk}$

\section{INTRODUCTION}

The acceleration of multi-MeV ion beams in the incidence of short-duration $\left(\tau_{1} \sim 1\right.$ $\mathrm{ps})$, high intensity $\left(\mathrm{I}>10^{18} \mathrm{~W} \cdot \mathrm{cm}^{-2}\right)$ laser pulses with metallic targets has been widely reported in the past decade ${ }^{1}$. Such ions beams exhibit a number of remarkable qualities including high brilliance, short burst duration, and an outstanding degree of laminarity $^{2}$, and their potential usefulness in a number of applications in science, technology and medicine has been frequently commented upon ${ }^{1,3}$. Their intrinsic divergence and broadband energy spectra, whilst hindering the development of their use in a number of applications ${ }^{4}$, is central to their unique prowess in diagnosing the processes occurring in intense laser-matter interactions.

In the proton probing (also referred to as the proton imaging/radiography) diagnostic, a laser-driven proton beam is combined with an energy-resolved detector

\footnotetext{
CP1153, Laser-Driven Relativistic Plasmas Applied to Science, Industry and Medicine - The $2^{\text {nd }}$ International Symposium edited by P. R. Bolton, S. V. Bulanov, and H. Daido

$\odot 2009$ American Institute of Physics 978-0-7354-0690-2/09/\$25.00
} 
to extract information on the processes occurring in a laser-matter interaction with $\mu \mathrm{m} \cdot \mathrm{ps}$ spatiotemporal resolution. The technique has already been applied successfully in the study of the interaction of short pulse lasers with both solid targets ${ }^{5}$ and underdense plasmas ${ }^{6}$; it has even elucidated the physical processes surrounding laserdriven ion acceleration itself ${ }^{7}$.

In this article, we will demonstrate how the proton probing diagnostic has been used to study a number of additional phenomena associated with the interaction of a short-pulse laser with a solid target. In the first experimental section (section A), we will describe the results of a study into interactions with $\sim 100 \mu \mathrm{m}$-diameter wire targets at $\sim 10^{19} \mathrm{~W} \mathrm{~cm}^{-2}$. We will see how the resultant target charging is highlytransient as the maximal E-fields measured at the wire surface rise to a peak magnitude $\sim 10^{10} \mathrm{~V} \cdot \mathrm{m}^{-1}$ before decaying to below measurable levels over a temporal window of $20 \mathrm{ps}$. Later, owing to the implementation of a novel experimental setup, the spread velocity of charge along the wire surface is measured to be $\sim c$. Theoretical reasoning and 2-dimensional (2-D) PIC simulations are used to infer that the wire charging observed is the result of the permanent escape to vacuum of a small fraction of the hot electrons accelerated at the focus of the interaction pulse. During the discharge phase, interesting filamentary structures $\sim 10-50 \mu \mathrm{m}$ in wavelength are observed to emanate from the wire normal to its surface. Pronounced magnetic features are also observed close to the interaction point.

In section $\mathrm{B}$, we will examine the results of a dataset concerning the interaction of a similarly-intense pulse with a variety of low-density foam targets. Laser-driven ion beams are most efficiently generated in the interaction of a pulse with a metallic foil. As stated, proton probing has been previously used to study ion acceleration from the surface of a laser-irradiated foil ${ }^{7}$; the exact nature of the processes occurring inside the target has remained elusive, however, since the high-density of metal makes this region inaccessible to probing. By using a low density foam target in place of a metallic foil the inside of the target can be made accessible to proton probing. The behaviour of the hot electrons generated by the interaction pulse inside the target foam is seen to depend on both target density and the level of doping applied. With the right combination of both parameters, electron focusing can occur; the wrong combination, however, results in the deleterious filamentation of the electron beam inside the target. The experimental foam-interaction results are shown to be in broad agreement with the output of a series of runs of 2-D LSP simulations.

\section{Laser-driven ion acceleration}

At intensities of greater than $10^{18} \mathrm{~W} \cdot \mathrm{cm}^{-2}$, the laser-driven acceleration of ion beams from the rear surface of thin foils is now generally accepted to occur by the Target Normal Sheath Acceleration (TNSA) mechanism ${ }^{7,8}$. In this framework, the proton acceleration pulse $\mathrm{CPA}_{1}$ drives a MA current of relativistic electrons through the target to the rear surface of the foil. The highly-relativistic nature of this electron beam means that, aside from some statistical scattering, it passes through the foil virtually unhindered. Whilst the most energetic of these electrons will escape to vacuum, before long, the initially-neutral target will develop a large positive electrostatic potential, and the bulk of the hot electrons generated by $\mathrm{CPA}_{1}$ will hence 
feel a restoring force towards the target. A Debye sheath of hot electrons will in turn form at the rear surface. The associated electric fields $\left(10^{11-12} \mathrm{~V} \cdot \mathrm{m}^{-1}\right)$ will lead to the rapid ionisation and acceleration of surface material ${ }^{7}$. The accelerated ions are predominantly protons due to the presence of hydrocarbon contaminants on the target surface 9 . The hot electron population proceeds to progressively transfer its energy to the expanding ion cloud until an equilibrium is reached, at which point the acceleration ceases and the beam proceeds as a quasineutral expansion into vacuum ${ }^{10}$. The resultant ion beam has a Boltzmann-like energy spectrum with a high-energy cutoff. The ion flux and cutoff energy depend on laser intensity and energy, and the target material and geometry. Cutoff proton energies $\sim 30 \mathrm{MeV}$ are routinely achievable at $10^{20} \mathrm{~W} \cdot \mathrm{cm}^{-2}$, whilst energies in excess of $50 \mathrm{MeV}$ have been observed at higher intensities.

\section{Proton probing}

Because they are composed of charged particles, TNSA proton beams are an extremely useful tool for the investigation of the electric and magnetic fields set up in an intense laser-matter interaction ${ }^{11}$. At a given distance from its source, the transverse density profile of a TNSA proton beam is largely uniform within its cone of divergence of half-aperture $\sim 20^{\circ}$. The presence of sufficiently large E- or B-fields in the interaction plasma of interest will then impinge transverse deflections on the probe protons, and the transverse density profile of the beam, $\mathbf{n}_{\mathbf{p}}$, will be perturbed accordingly by $\delta \mathbf{n}_{\mathbf{p}}$. These modulations in $\mathbf{n}_{\mathbf{p}}$ hence serve as a measure of the electric and/or magnetic fields in the region of the interaction.

Whilst the laser-driven probe proton beam is globally neutralized by the cloud of co-moving electrons, the scale lengths of the electric and magnetic fields under investigation are significantly shorter than the local Debye length of the quasineutral beam at the interaction region so that, whilst probe protons don't repel one another, they may still be deflected by the action of external E- and B-fields at the interaction region $^{7,11}$.

Suppose the proton source can be approximated to lie at the point on the foil opposite to the point at which the acceleration pulse $\mathrm{CPA}_{1}$ is incident, and that the interaction plasma of interest and transverse proton detector lie at distances of $d$ and $D$ from this source, respectively. The magnification of the interaction region at the detector will then be given by $M=D / d$.

The proton detector usually takes the form of a series of layers of radiochromic films ${ }^{12}$ (RCFs), commonly referred to as an RCF stack. RCF is an absolutelycalibrated dosimetry medium that is sensitive to ionising radiation. An unexposed $\mathrm{RCF}$ is clear, but will turn a progressively deeper shade of blue as more radiation (or a more intense proton dose) is applied to it. As stated, laser-driven protons are intrinsically broadband. The fact that protons travelling through a solid will deposit most of their energy near the end of their range (in the so-called Bragg Peak) means that, when an RCF stack is employed as a proton detector, the distance into the pack to which a proton of a given energy will travel before it is stopped can be estimated, so that each layer can effectively be associated to a proton energy, $\mathrm{E}_{\mathrm{p}}$. More energetic protons will travel to deeper layers in the pack, and vice versa. This intrinsic energy 
resolution of the pack translates to temporal resolution in the diagnostic, however, so that the probing time that may be associated to a given layer may be computed by

$$
t_{\text {probe }}=d\left(2 E_{p} / m_{p}\right)
$$

where $m_{p}$ is the proton mass. A typical proton cutoff energy of $30 \mathrm{MeV}$ and sourceinteraction distance of $d=3 \mathrm{~mm}$ hence facilitates probing of the interaction region over a temporal window $\sim 50 \mathrm{ps}$ with ps resolution.

\section{Particle tracing simulations}

The spatial distribution and temporal evolution of the electric and magnetic fields set up in the wake of a single intense laser-matter interaction may be inferred by comparing the experimental RCF stack data with the results of a 3-D particle tracer. The simulation code qTrace was developed by our group to simulate the propagation of a proton beam through a field-containing interaction box (FIGURE 1).

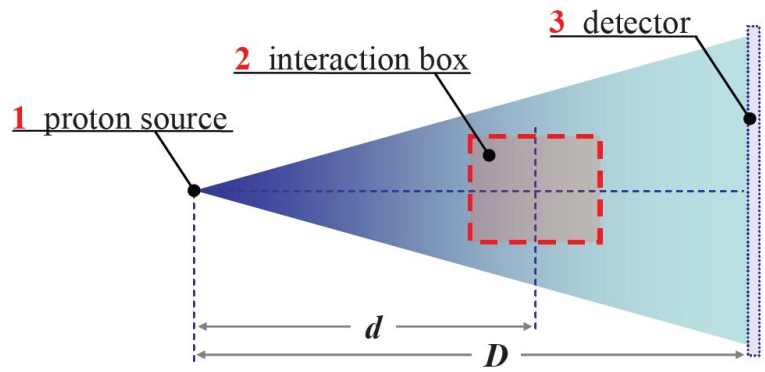

FIGURE 1. The three basic steps involved in the running of a particle tracing simulation. The divergent proton beam is emitted from the source (1) then modulated by the deflecting fields of the interaction region (2) before impacting upon the transverse detector (3).

The divergent probe beam is produced at the source in a Maxwell-Boltzmann energy distribution according the specifications of the user with regard to temperature, total number of protons etc. The traversal of each proton through the interaction box is in turn dealt with by splitting its motion in this region into a discrete number of steps. As a proton bridges the gap between two adjacent traversal steps, the velocity change it experiences may be calculated by solution of the Lorentz equation:

$$
\delta \mathbf{v}_{p}=\frac{e}{m_{p}} \int_{t-\delta t / 2}^{t+\delta t / 2}\left(\mathbf{E}+\mathbf{v}_{p} \times \mathbf{B}\right) \delta t
$$

where $e$ is the elementary charge and $\delta$ is the time taken to move from one step to the next. In the absence of fields inside the interaction region, the transverse proton density function $\mathbf{n}_{\mathbf{p}}$ recorded at the detector is uniform. Correspondingly, the presence of E- and/or B-fields inside the interaction box may impose a modulation on $\mathbf{n}_{\mathbf{p}}$. Hence, by repeatedly prescribing $\mathbf{E}(\mathrm{t})$ and $\mathbf{B}(\mathrm{t})$ until a best-match is obtained between 
experiment and simulation, the evolution and spatial distribution of the fields inside the interaction box is inferred.

The effects of scattering and energy loss inside the target may also be modelled in the particle tracer. Whilst energy loss is of minimal significance in the traversal of multi-MeV protons through relatively thick $(\sim 100 \mu \mathrm{m})$ metallic targets, scattering can be important. The Monte-Carlo ion stopping and scattering program $\operatorname{SRIM}^{13}$ was hence employed to enable calculation of the degree of scattering suffered by protons passing through various thicknesses of target material as a function of energy.

\section{The laser system}

The experiment was performed on the Vulcan laser system at the Rutherford Appleton Laboratory ${ }^{14}$. In Target Area Petawatt, operating at a central wavelength of $1.054 \mu \mathrm{m}$, the main beamline of Vulcan is capable of delivering $500 \mathrm{~J}$ in $500 \mathrm{fs}$. Focusing of the $60 \mathrm{~cm}$-diameter main beam is performed via a $1 \mathrm{~m}$ off-axis parabola so that intensities approaching $10^{21} \mathrm{~W} \cdot \mathrm{cm}^{-2}$ may be achieved at best focus. Some $5 \%$ of the beam energy was optically diverted inside the target chamber to provide the interaction pulse $\left(\mathrm{CPA}_{2}\right)$, whilst the remainder provided the proton acceleration pulse $\left(\mathrm{CPA}_{1}\right)$. Cutoff energies of $40 \mathrm{MeV}$ were observed in the probe proton beam so that the interaction could typically be observed over a 50 ps time window with ps resolution. Note that both the spatial and temporal resolution of the proton imaging technique improve as energy increases (with increasing velocity, probe protons will suffer smaller deflections and be in the region of the target for shorter times).

\section{SECTION A: WIRE INTERACTIONS}

The study of laser-wire interactions can be fruitful in yielding information on lasersolid interactions in general. The fact that the target is an essentially 1-D object makes the laser-wire interaction in some ways easier to investigate and interpret than the 2-D laser-foil interaction. The incidence of a short-pulse laser with a cone-wire target has been previously studied in a Fast Ignition context ${ }^{15}$; so too has the possibility of harnessing the large currents generated in the laser-wire interaction in the production of fast rise-time $Z$-pinches ${ }^{16}$.

\section{Observation of wire charge-discharge cycle}

In the present research, the proton probing diagnostic has been used to investigate the incidence of a $30 \mathrm{~J}, 3 \times 10^{19} \mathrm{~W} \cdot \mathrm{cm}^{-2}$, ps-duration laser pulse $\left(\mathrm{CPA}_{2}\right)$ with a 125 $\mu \mathrm{m}$-diameter gold wire (FIGURE 2). The probe beam is generated in the interaction of a $4 \times 10^{20} \mathrm{~W} \cdot \mathrm{cm}^{-2}$ pulse $\left(\mathrm{CPA}_{1}\right)$ with a gold foil. The probe beam zero-level is defined as the horizontal level at which the protons emitted from the source have no vertical component of velocity. As shown in the figure, the probe beam zero-level lies some $600 \mu \mathrm{m}$ above the $\mathrm{CPA}_{2}$ interaction point. This ensures that the processes occurring in the bulk target are probed fully as opposed to the already welldocumented TNSA behaviour close to the $\mathrm{CPA}_{2}$ interaction point. 


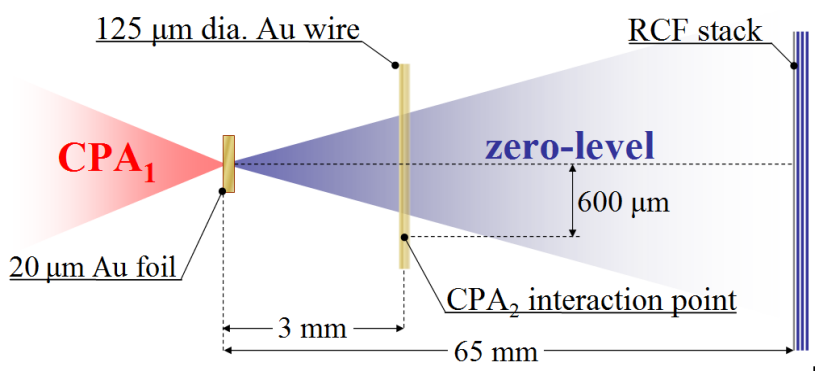

FIGURE 2. Experimental setup for investigation into the interaction of $\mathrm{CPA}_{2}$ with a metallic wire via proton probing. $\mathrm{CPA}_{2}$ strikes into the page.

The wire will develop a positive potential in the wake of the $\mathrm{CPA}_{2}$ interaction as electrons are accelerated away from the target in the radial direction. By the skin effect, positive charge will tend to be restricted to a thin surface layer on the wire; the bulk of the laser-accelerated hot electrons which fail to escape to vacuum will be highly-relativistic in the initial phase, however, and may form an extensive cloud around the target. Cylindrical $(\mathrm{r}, \theta, \mathrm{z})$ geometry is appropriate for the mathematical modeling of this problem. If it is assumed that the negative charge density outside the wire is low compared to the positive charge density inside, then outside the wire, the magnitude of the radial E-field may be approximated by

$$
E(r) \approx E_{\text {peak }}\left(r_{w} / r\right)
$$

where $r$ is the radial distance from the wire axis, $E_{\text {peak }}$ is the magnitude of the E-field at the wire surface, and $r_{w}$ is the wire radius. Inside the wire, meanwhile, the E-field will decay rapidly, falling to $1 / e$ of $E_{\text {peak }}$ within a skin depth of the wire surface, where $e$ is the base of the natural logarithm. By Ampère's Law, the presence of a non-zero current on the target surface will lead to the development of a related azimuthal magnetic field, $\mathrm{B}_{\theta}$, in the region of the wire. It can be shown via the use of the particle tracer, however, that so long as the maximum magnitude of $\mathrm{B}_{\theta}$ does not exceed $100 \mathrm{~T}$, then the deflection pattern observed at the RCF can be assumed to be the result of the radial E-field surrounding the wire alone. The validity of this assumption can be verified a posteriori.

Samples from the experimental RCF dataset are displayed in FIGURE 3 below. $\mathrm{CPA}_{2}$ is incident from the bottom-right, whilst the wire image is clearly visible as the vertical band of proton depletion running down the centre of each image. The $\mathbf{n}_{\mathbf{p}}$ behaviour at the zero-level is unperturbed in image (i). By image (iii), however, $4 \mathrm{ps}$ after the $\mathrm{CPA}_{2}$ interaction time, sharp build-ups in $\mathbf{n}_{\mathbf{p}}$ are visible on either side of the wire shadow as a pair of dark blue lines symmetrical about the wire axis. This feature is caused by the deflection of centrally-directed probe protons away from the wire. By image (iv), these $\mathbf{n}_{\mathbf{p}}$ pile-ups are clearly visible along the length of the wire. At later times, however, the maximum extent of these $\mathbf{n}_{\mathbf{p}}$ pile-ups appears to decrease with time, indicating that the wire is beginning to discharge. 


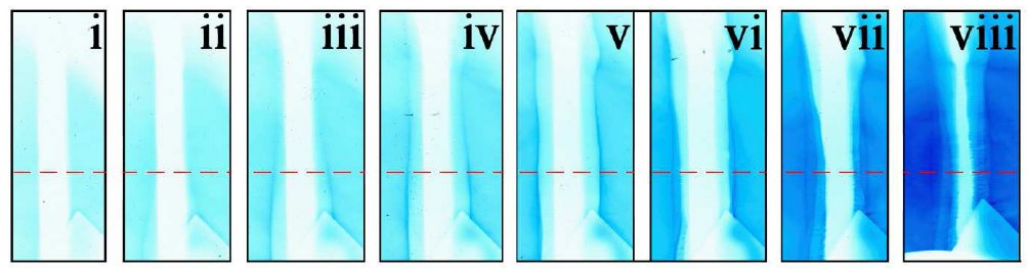

FIGURE 3. Samples of RCF data describing the incidence of $\mathrm{CPA}_{2}$ with a $125 \mu \mathrm{m}$-diameter gold wire. The zero-level of the probe beam is denoted by the horizontal dashed red line. The $\mathrm{CPA}_{2}$ interaction plasma is visible to the bottom-right of each image as a circular area of proton depletion (a white region). Relative to the time of arrival of the $\mathrm{CPA}_{2}$ interaction pulse, the probing times associated to each RCF layer are, from (i)-(viii): $0,2,4,5,7,10,17$, and $25 \mathrm{ps}$, respectively.

The particle tracer was repeatedly run with different values of $E_{\text {peak }}$ for each RCF layer in the dataset until a best-match was obtained between the experimental and simulated zero-level functions for $\mathbf{n}_{\mathbf{p}}$. In this way, a complete temporal picture of the rise and decay of the radial E-field at the zero-level was produced. The maximum value of E-peak was hence determined to be $8 \times 10^{9} \mathrm{~V} \cdot \mathrm{m}^{-1}$ (see FIGURE 4). The decay of the measured E-field (and its related current) is facilitated by (i) the even spreading of charge over the wire, (ii) the recombination of hot electrons with the positively-charged wire plasma, and ultimately, (iii) by the flow of electrons from the grounded target mount.

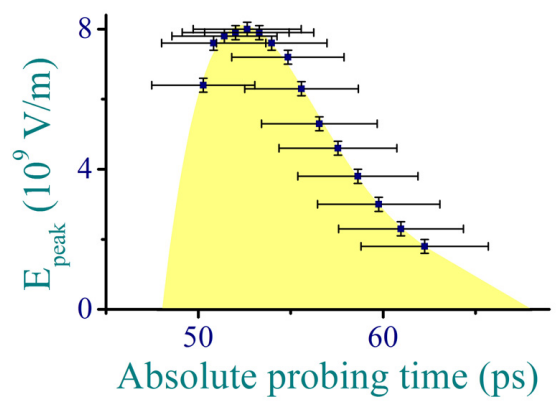

FIGURE 4. Description of the growth and decay of the radial E-field at the wire surface at the zerolevel. The 'absolute probing time' referred to is relative to the acceleration of the probe beam by $\mathrm{CPA}_{1}$.

The horizontal error bars were computed by consideration of the transit time of a probe proton of a given energy through the significantly-deflecting field-containing region surrounding the wire.

\section{Ultrafast current propagation along wire surface}

Whilst the result displayed in FIGURE 4 constitutes a well-resolved picture of the charging and subsequent discharging of the wire over a temporal window of $20 \mathrm{ps}$ in the wake of the $\mathrm{CPA}_{2}$ interaction, the actual spread of charge along the wire surface from the interaction point is still not resolved. The reason for this is that each RCF layer in this dataset corresponds effectively to a discreet point in time. By placing the interaction wire at an angle to the vertical, however, we can resolve the flow of ultrafast currents along the wire surface (see FIGURE 5a). In the shot depicted, the wire angle was $\theta=30^{\circ}$. As stated previously, the bulk of the signal on a given RCF 
layer can be attributed to protons of a given energy/velocity. When the wire is angled toward the proton source, then, the protons emitted at different elevation angles $\alpha$ relative the zero-level will probe different points along the wire at different times, so that, effectively, continuous observation of the target is provided. As a result, this experimental setup allows for the resolution of the propagation of a charging front along the wire at $v_{f}=(0.95 \pm 0.05) c$ (FIGURE $5 \mathrm{~b}$ ).
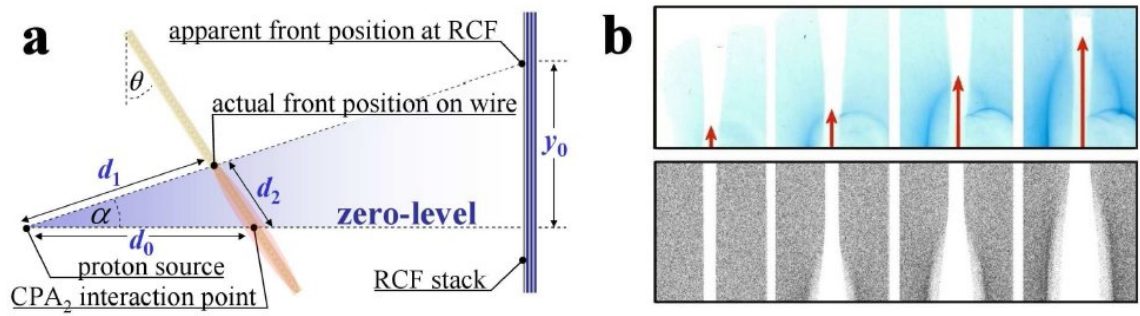

FIGURE 5. (a) Experimental setup for angled wire shot. $\mathrm{CPA}_{2}$ strikes into the page. (b) Experimental (top) and simulated (bottom) RCF data displaying the propagation of the charging front away from the interaction point. The front motion is indicated by the red arrows. The $\mathrm{CPA}_{2}$ interaction plasma is visible to the bottom-right of each experimental image. The velocity at which the charging front moves along the wire is measured to be $v_{f}=(0.95 \pm 0.05) c$.

\section{Electron escape}

The observation of the propagation of this field front away from the $\mathrm{CPA}_{2}$ interaction point at $v_{f} \sim c$ allows the previous result, in describing the evolution of the magnitude of the E-field at the wire surface, $E_{\text {peak }}(t)$, to be interpreted as resulting from the flow of a transient current past the zero-level, the magnitude of which can be shown to be given by

$$
I(t)=2 \pi \varepsilon_{0} r_{w} v_{f} E_{p e a k}(t)
$$

where $\varepsilon_{0}$ is the permittivity of free space. The wire current at the zero-level at the peak of the charging cycle is hence calculated to be $\sim 10^{4} \mathrm{~A}$. Integration of $I(t)$ over time reveals the total number of electrons moving downwards past the zero-level to be $\sim 10^{11}$. Theoretical considerations ${ }^{17}$ reveal that this figure is consistent with the total number of hot electrons which are expected to escape to vacuum in the wake of the $\mathrm{CPA}_{2}$ interaction.

In our interpretation, the permanent escape to vacuum of $\sim 1 \%$ of the laseraccelerated hot electrons generates a large positive potential which leads to the antenna-like propagation of an electromagnetic disturbance away from the interaction region which propagates freely in vacuum at $\sim c$ but only penetrates the skin layer of the target, drawing a return electron current as it does so. This interpretation is supported by the results of 2-D PIC simulations (FIGURE 6). The pulse is incident from the left at the front surface of the target, a region not visible in the figure. Two current $\left(J_{y}\right)$ fronts are visible: an outer one travelling at $c$, and an inner, more slowlymoving one which is consistent with the spread of hot electrons up the wire. 

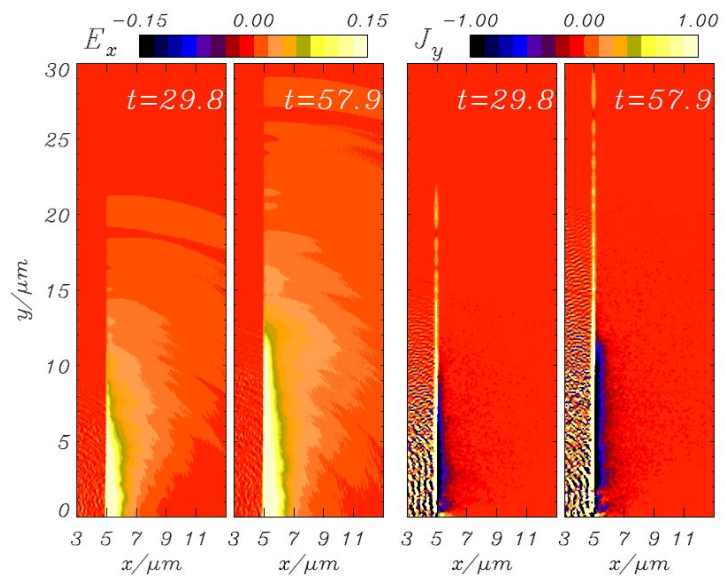

FIGURE 6. PIC simulation results showing the contours of $E_{x}$ and $J_{y}$ in the region around the rear target. Two different times (in fs) are depicted relative to the arrival of the pulse from the left at the front target surface (not pictured). The rear surface is at $x=5 . E_{x}$ is in units of $3.05 \times 10^{8} \mathrm{~V} \cdot \mathrm{cm}^{-1}$ and $J_{y}$, in units of $4.8 \times 10^{13} \mathrm{~A} \cdot \mathrm{cm}^{-2}$.

\section{Filamentary structures}

Fine-scale filamentary structures are seen to develop during the wire discharge phase (FIGURE 7). They appear to emanate normal to the wire surface, and persist for relatively long timescales (tens of ps), suggesting that they may be sustained by the presence of quasi-static magnetic fields. The onset of these periodic striations normally begins some $10 \mathrm{ps}$ after the arrival of the ps-duration $\mathrm{CPA}_{2}$ interaction pulse, and the spatial wavelength of their periodicity is between 10 and $50 \mu \mathrm{m}$, depending on the wire material and interaction conditions. It is likely that these filaments are seeded as the hot electrons returning from vacuum interact with the relatively cold wire plasma ${ }^{18}$, but analysis of this dataset is ongoing and will be the subject of a future publication.

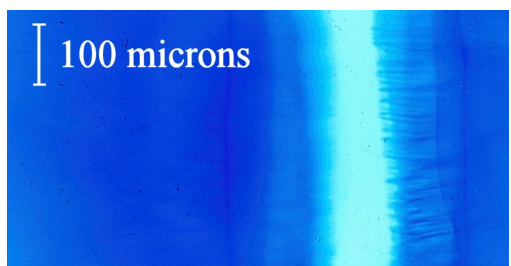

FIGURE 7. Magnified region of FIGURE 3(viii). The RCF layer depicted corresponds to a probing time $25 \mathrm{ps}$ after the $\mathrm{CPA}_{2}$ interaction. Filamentary structures are particularly-visible to the right of the wire shadow. The zero-level runs horizontally through the centre of the image. 


\section{SECTION B: FOAM INTERACTIONS}

The experimental setup depicted in FIGURE 2 is again employed for the proton probing of the interaction of a $2 \times 10^{19} \mathrm{~W} \cdot \mathrm{cm}^{-2} \mathrm{CPA}_{2}$ pulse with a foam target. The laser-foam interaction under investigation is described in FIGURE 8 below. With a diameter of $1.5 \mathrm{~mm}$ and a thickness of $300 \mu \mathrm{m}$, the foam itself is effectively a laminar target. The electron propagation under investigation can therefore be assumed to occur only in the plane transverse to that of the proton probe, a fact which greatly simplifies the interpretation of the experimental data. Shots were performed using a variety of foam densities (from 20 to $200 \mathrm{mg} \cdot \mathrm{cc}^{-1}$ ) and dopant levels. The chemical formula of the pure foam used is $\mathrm{C}_{15} \mathrm{H}_{20} \mathrm{O}_{6}$. A $75 \mathrm{~nm}$-thick gold coating was applied to the interaction edge of each foam so that, regardless of the foam density or level of doping used, the parameters of the hot electron beam accelerated into the target could be assumed to remain relatively constant.

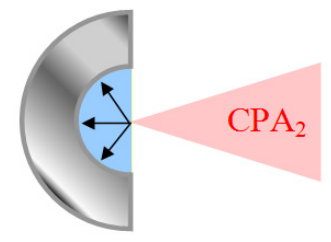

FIGURE 8. Schematic describing the interaction of $\mathrm{CPA}_{2}$ with a foam target. The foam is held inside a metallic half-washer. The propagation of hot electrons through the foam is indicated by the arrows.

\section{Possible observation of electric inhibition}

Samples of the RCF data obtained in the interaction of $\mathrm{CPA}_{2}$ with a $100 \mathrm{mg} \cdot \mathrm{cc}^{-1}$ foam are shown in FIGURE 9. Image (a) shows two dark lines of proton density pileup separated by an angle of $60^{\circ}$. This effect is witnessed to commence 14 ps prior to the intensity peak of the $\mathrm{CPA}_{2}$ pulse. At this time, the intensity of the pulse is expected to be $\sim 10^{16} \mathrm{~W} \cdot \mathrm{cm}^{-2}$, corresponding to a hot electron temperature $\sim 50 \mathrm{keV}$. Such features may be related to the electric and magnetic fields associated with the injection of a divergent electron beam into the target. Preliminary analysis using 3-D particle tracing simulations support the presence of a diverging electron cone. At later times the dark lines are observed to evolve into white channels (regions of low $\mathbf{n}_{\mathbf{p}}$, see FIGURE 9d), possible evidence of the presence of a persisting magnetic field.

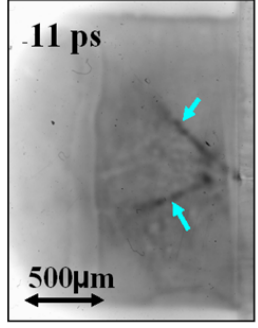

(a)

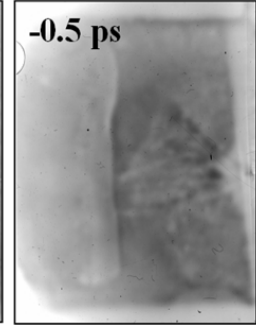

(b)

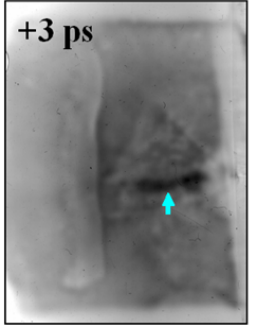

(c)

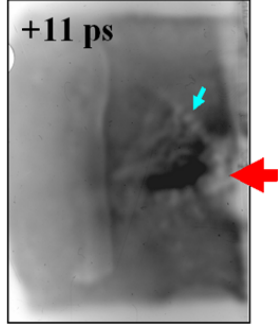

(d)

FIGURE 9. RCF images associated to four different times relative to the peak of the $\mathrm{CPA}_{2}$ interaction pulse. The pulse is incident from the right. 
As is visible in images (c) and (d), in the immediate aftermath of the peak of the interaction pulse, a pronounced, dark horizontal feature is observed in the direction of laser propagation, suggesting the presence of a very strong electric field. This may correspond to a strong, highly collimated electron jet injected into the target as suggested by previous observations ${ }^{19}$. It has been suggested that initial electric inhibition results in the formation of a low resistivity channel due to Ohmic heating, and the resultant magnetic field generated at the edge of the electron beam channel inside the target leads to strong collimation ${ }^{20}$. The limited extent of the dark feature may correspond to a limited penetration depth of the injected electrons, possible evidence of the occurrence of electric inhibition at low density ${ }^{21}$.

\section{Filamentation and instability growth}

Relativistic electron beam propagation can be largely affected by plasma instabilities. In the context of Fast Ignition, the Weibel instability ${ }^{22}$ can play a significant role since it is detrimental to the coupling of the electron energy to the compressed fusion pellet ${ }^{23}$.

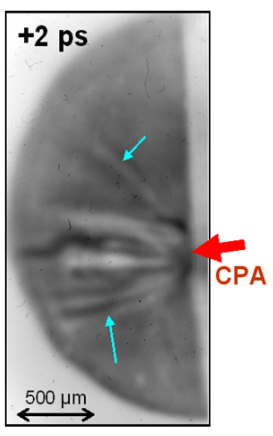

(a)

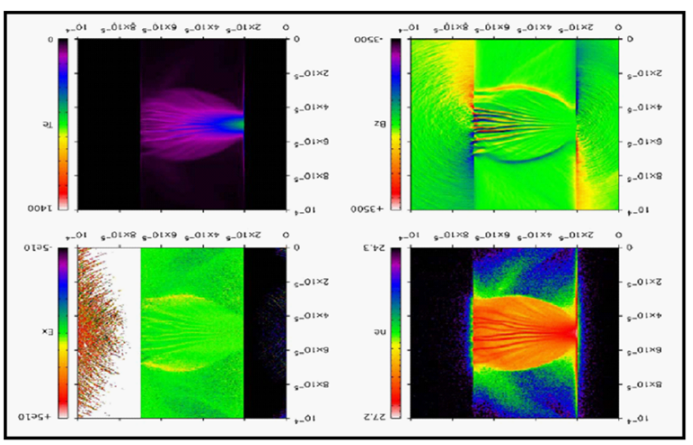

(b)

FIGURE 10. (a) RCF data showing filamentation inside a $50 \mathrm{mg} \cdot \mathrm{cc}^{-1}, 30 \% \mathrm{Br}$-doped foam.

(b) Comparison with the results of an LSP simulation ${ }^{24}$ matched to the experimental parameters. The distributions of temperature, magnetic field strength, and hot electron density are shown.

FIGURE 10a shows one of the RCF images obtained using a Br-doped foam target. Very strong filamentation is observed. Filaments are more prominent along the laser propagation direction, as is consistent with the hot electron current being higher in this region. This will result in strong current pinching as the return current becomes insufficient to balance the injected relativistic current. The filaments along the laser direction appear white as the probe protons are expelled almost completely from this region. This may be due to the presence of large magnetic fields around the filaments. The filamented transport of $\mathrm{MeV}$ electrons in low-conductivity material is consistent with previous, indirect observations ${ }^{25}$.

Whilst analysis of this data is in its preliminary stages, the RCF images describing the transit of laser-driven hot electrons through dense material are interesting as it 
stands, and the data goes at least some way to furthering our understanding of the processes governing their production and transport characteristics.

\section{ACKNOWLEDGMENTS}

This research was supported by: EPSRC grants P/E048668/1, EP/E035728/1 and EP/C003586/1; DFG Nos. TR18 and GK1203; the British Council Alliance Programme; QUB Internationalisation Funds; a DEL/CAST scholarship part-funded by AWE plc; the Direct Access Scheme to the Central Laser Facility; and finally, by a Dorothy Hodgkin Postgraduate Award funded by EPSRC and Andor Technology. The support of the staff of the Central Laser Facility at RAL is gratefully acknowledged.

\section{REFERENCES}

1. M. Borghesi et al., Fus. Sci. Tech. 49, 412 (2006)

2. T. E. Cowan et al., Phys. Rev. Lett. 92, 204801 (2004)

3. S. V. Bulanov et al., Phys. Lett. A 299, 240 (2002)

4. U. Linz and J. Alonso, Phys. Rev. ST Accel. Beams 10, 094801 (2007)

5. M. Borghesi et al., Appl. Phys. Lett. 82, 1529 (2003)

6. S. Kar et al., New J. Phys. 9, 402 (2007)

7. L. Romagnani et al., Phys. Rev. Lett. 95, 195001 (2005)

8. R. A. Snavely et al., Phys. Rev. Lett. 85, 2945 (2000)

9. S. J. Gitomer et al., Phys. Fluids 29, 2679 (1986)

10. P. Mora, Phys. Rev. Lett. E 72, 056401 (2005)

11. L. Romagnani et al., Laser Part. Beams 26, 241 (2008)

12. http://www.gafchromic.com

13. http://www.srim.org

14. C. Danson et al., Nucl. Fusion 44, S239 (2004)

15. J. S. Green et al., Nature Phys. 3, 853 (2007); R. Kodama et al., Nature 432, 1005 (2004)

16. F. N. Beg et al., Phys. Rev. Lett. 92, 095001 (2004)

17. K. Quinn et al., Eur Phys J D, (2009), DOI: 10.1140/epjd/e2009-00079-7

18. M. G. Haines, Phys. Rev. Lett. 47, 917 (1981)

19. L. Gremillet et al., Phys. Rev. Lett. 83, 5015 (1999)

20. J. R. Davies et al., Phys. Rev. E 59, 6302 (1999)

21. F. Pisani et al., Phys. Rev. E 62, R5927 (2000)

22. E. S. Weibel, Phys. Rev. Lett. 2, 83 (1959)

23. M. Honda et al., Phys. Rev. Lett. 85, 2128 (2000)

24. R. G. Evans, High Energy Density Physics 2, 35 (2006)

25. J. Fuchs et al., Phys. Rev. Lett. 91, 225002 (2003) 